\title{
Research on The Realization and The Correction Method of Velocity Interpolation Algorithm for The Quadratic Curve
}

\author{
Chi Tang ${ }^{\mathrm{a}}$, Ying $\mathrm{Li}^{\mathrm{b}}$ \\ School of Mechanical and Power Engineering, East China University of Science and Technology, \\ Shanghai 200000, China \\ a tangchi023@163.com , b yingli163@sina.com
}

Keywords: velocity interpolation; stepper motor; VC++; quadratic curve

\begin{abstract}
Aiming at the problem of low efficiency and low accuracy of traditional interpolation algorithms, a new numerical control interpolation method-velocity interpolation, is presented in this paper. The realization steps of the quadratic curve in the numerical control system are described. Through the analysis of the error caused by the error caused by the stepper motor in the stepper motor control system, a correction method is proposed to improve the machining accuracy. The simulation program is developed by $\mathrm{VC}++$ to simulate the process of the quadratic curve, which is the representative of a parabola, and the feasibility of the modified method is verified.
\end{abstract}

\section{Introduction}

For NC milling machine, the core of digital control is to control the relative motion between the tool and the workpiece. In order to process the workpiece with a given profile, it is required that the workpiece and the cutting tool can be coordinated. Coordination is essentially the movement sequence, displacement, direction and speed of each axis in the process of the linkage, which is the interpolation. The high accuracy of the interpolation algorithm determines the quality of the workpiece, and the operation rate will improve the processing efficiency.

The traditional interpolation algorithms are mainly divided into two categories, namely the pulse increment interpolation ${ }^{[1]}$ and the data sampling interpolation ${ }^{[2]}$. The basic principle of them is the use of a line or arc (mainly linear and circular nodes) continuous approximation theory of curves between segments by piecewise fitting way to complete the processing curve. Therefore, on the one hand, the traditional algorithm is to deal with complex curve will lead to such a large amount of data, the overall processing time is longer and the efficiency is low; on the other hand, the curve between the nodes caused by a lack of continuity, the processing precision is not high ${ }^{[3]}$.

In view of the above problems, this paper introduces a method of velocity interpolation. The quadratic curve function is decomposed into $\mathrm{X}, \mathrm{Y}$ two directions of the speed function, and according to the two speed function equation of time interpolation control processing trajectory. Meanwhile, the machining error caused by step out of stepper motor is analyzed, and a correction method is proposed, and the simulation program is developed by $\mathrm{VC}++$.

\section{Velocity interpolation principle}

The known quadratic curve trajectory function expression for $y=f(x)$, each $x i$ has a corresponding determined $y i$ and its parameter equation can be written as:

$$
x=s(t), y=g(t)
$$

The first-order derivative of formula one, we can get the velocity equation:

$$
x^{\prime}=s^{\prime}(t)=V x(t), y^{\prime}=g^{\prime}(t)=V y(t) ;
$$

The point ( $x i, y i)$ incremental approximation in increments $\Delta t$ of the time $t$ value can be got:

$$
\Delta x i=V x(t) \Delta t, \Delta y i=V y(t) \Delta t
$$

The $V x(t), V y(t)$ respective the $\mathrm{X}$ axis and $\mathrm{Y}$ axis velocity of the point $((x i, y i)$ in time $t$.For the given quadratic curve trajectory, the initial point is $(x 0, y 0)$, the termination point is $(x, y)$.The 
trajectory is divided into $n$ nodes, and the processing time is $t$, the movement time between adjacent nodes is $\Delta t=t / n$, the following formula is:

$$
x=x 0+\sum V x \Delta t=x 0+\Delta t \sum V x, y=y 0+\sum V y \Delta t=y 0+\Delta t \sum V y
$$

From the above equation, under the condition of constant time interval $\Delta t$, the node velocity is variable parameter, the velocity varies with different node position and time. So if the time $\Delta t$ is given, whole trajectory can be controlled by planning the speed $V x$ and $V y$ of each node ${ }^{[4]}$.

In summary, the processes of speed interpolation in CNC machine tools can be divided into the following steps:

1.According to the expression of the given machining path function, the function expression of the velocity of the trajectory in the $\mathrm{X}$ and $\mathrm{Y}$ directions is calculated;

2.The machining trajectory curve is divided into n parts, and determine the processing time $t$, each node velocity ( $V x i$, Vyi) is calculated by algebraic formulal;

3.According to the relevant parameters of the numerical control system, the speed signal is converted into the corresponding motor control;

4.The parameter $\Delta t=t / n$ is used as the time interval, pulse velocity signal will be transmitted to $\mathrm{X}$, Y direction of the motor to achieve multi-axis linkage, the realization of the approximation of the theoretical trajectory also can be completed.

\section{Error analysis and solution}

The approximation degree between the processing trajectory and the theoretical trajectory depend on the displacement increment in each time, it means that the approximation degree coincides with deviation degree between the actual position and the theoretical position after each velocity interpolation. In the stepper NC system, the speed of the stepper motor can be changed according to the speed of the signal. The accuracy of the process can be affected by the instability of the driving current, the change of the speed of the rotating speed, the torque caused by the instability of the driving current ${ }^{[5]}$. The step out of stepper motor includes lost step and more step, lost step means that the rotor forward step number is less than the number of pulses; In contrast, more step means that the rotor forward step number is more than the number of pulses. As shown in Fig.1, the solid line shows the theoretical trajectory, and the dotted line for the actual trajectory. In the process of velocity interpolation, simultaneously more step of two stepper motors, make the $\mathrm{X} 1$ '-X0>X1-X0, $\mathrm{Y} 1$ '- $\mathrm{Y} 0>\mathrm{Y} 1-\mathrm{Y} 0$, resulting in the actual point in the top right of the theoretical point. In the second stage, simultaneously lost step of two stepper motors make the $\mathrm{X} 2-\mathrm{X} 1>\mathrm{X} 2$ '-X1', Y2-Y1>X2'-X1, resulting in the actual point in the lower left of the theoretical point.

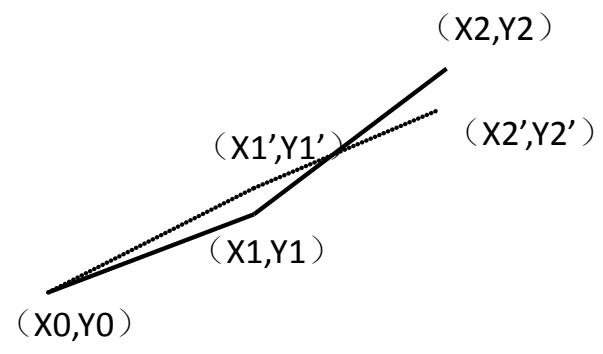

Fig. 1 Theoretical Trajectory and Actual Trajectory

The error caused by step out of the stepper motor is uncertainty, the starting point of every interpolation is a last interpolation error position. If the displacement increment according to the plan, it will produce error superposition of the two aspects, causing machining instability and affect the processing accuracy.

A control scheme is proposed to improve the processing accuracy in this paper. The results of every interpolation by stepper motor are feedback and compared with the theoretical nodes to obtain 
a deviation value in $\mathrm{X}, \mathrm{Y}$ direction. The deviation value can be corrected by the next interpolation, so as to reduce the deviation degree between actual point and theoretical point. As shown in Fig. 2, the solid line shows the theoretical trajectory, and the dotted line for the correction path, when processing path reach the first point, and it produce the errors: $\Delta \mathrm{X} 1=\mathrm{X} 1$ "'-X $0, \Delta \mathrm{Y} 1=\mathrm{Y} 1$ " $-\mathrm{Y} 0$. So the next time interpolation, the given displacement increment will be corrected, the correction values equal to $t$ The difference between the original displacement increment and the previous error which is $\Delta \mathrm{X} 1$ and $\Delta \mathrm{Y} 1$. Each interpolation behind will have a comparison between the actual value and the theoretical value, to provide a correction value for the next interpolation. The correction displacement increment still exist error due to the stepper motor's step error, but by comparing the figures 1 and 2, the deviation degree is reduced, the processing trajectory is reduced, and the stability is improved.

\section{$(\mathrm{X} 2, \mathrm{Y} 2)$}

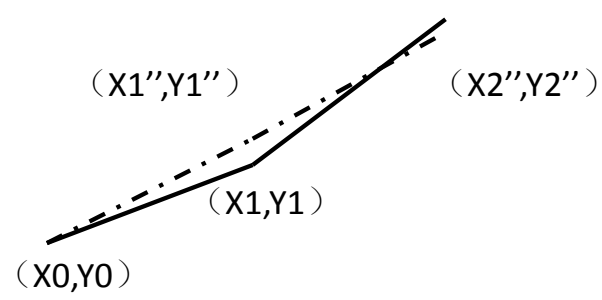

Fig.2 Theoretical Trajectory and Correction Trajectory

In order to evaluate the degree of deviation, this paper uses a similar standard deviation, which is the open root value of the average number of the distance square between the actual point and theoretical point, the formula is as follows:

$$
S=\sqrt{\frac{\sum_{k=1}^{n}\left[(X i \prime-X i)^{2}+(Y i \prime-Y i)^{2}\right]}{n}}
$$

In the formula, $s$ is the numerical value of the deviation degree, $X i$ 'and $Y i$ ' is the horizontal and vertical coordinates of the actual points, $X i$ and $Y i$ are the horizontal and vertical coordinates of the theoretical points, and $n$ is the number of setting nodes.

\section{The realization of velocity interpolation processing and the correction trajectory simulation based on $\mathrm{VC}++$}

In order to verify the velocity interpolation principle and the feasibility of the control scheme, the simulation program is developed by $\mathrm{VC}++{ }^{[6]}$.

In this program, parabola as a typical two curves is on research. The processing path of velocity interpolation and the comparison of the error graph and the correction graph are simulated in the drawing interface. At the same time, the process data of various cases are processed, and the standard deviation of the error processing path and correction process path and maximum deviation values are obtained after the end of the simulation. The effect of the control method is verified by graphics and data, The whole simulation software is shown in Fig.3. 


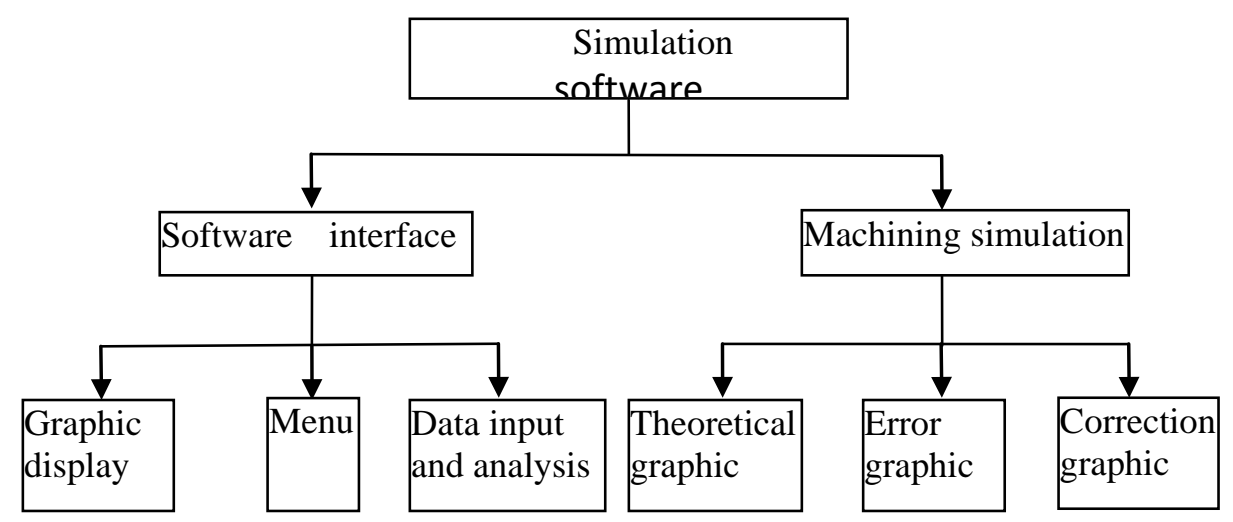

Fig.3 Simulation Software

Processing simulation is mainly the simulation of the error and the correction of the two conditions of the processing trajectory.

Drawing error graphics code:

void CSpdView::OnError() // Draw the error parabola.

\{

float $\mathrm{xi}=0, \mathrm{yi}=0$;

for(int $\mathrm{i}=1 ; \mathrm{i}<\mathrm{pDoc}->\mathrm{JIE} ; \mathrm{i}++)$

$\{$ float $\mathrm{xe}=10+10 *(-1+\operatorname{rand}() \% 3) * 0.05$,

ye $=\left(\right.$ pDoc- $>$ PARM $*{ }^{*} * \mathrm{i} * 100-$ pDoc- $>$ PARM $\left.*(\mathrm{i}-1) *(\mathrm{i}-1) * 100\right)$

$+($ pDoc->PARM $* i * i * 100-$ pDoc- $>$ PARM $*(i-1) *(i-1) * 100) *(-1+\operatorname{rand}() \% 3) * 0.05$;

// The error displacement increment each time

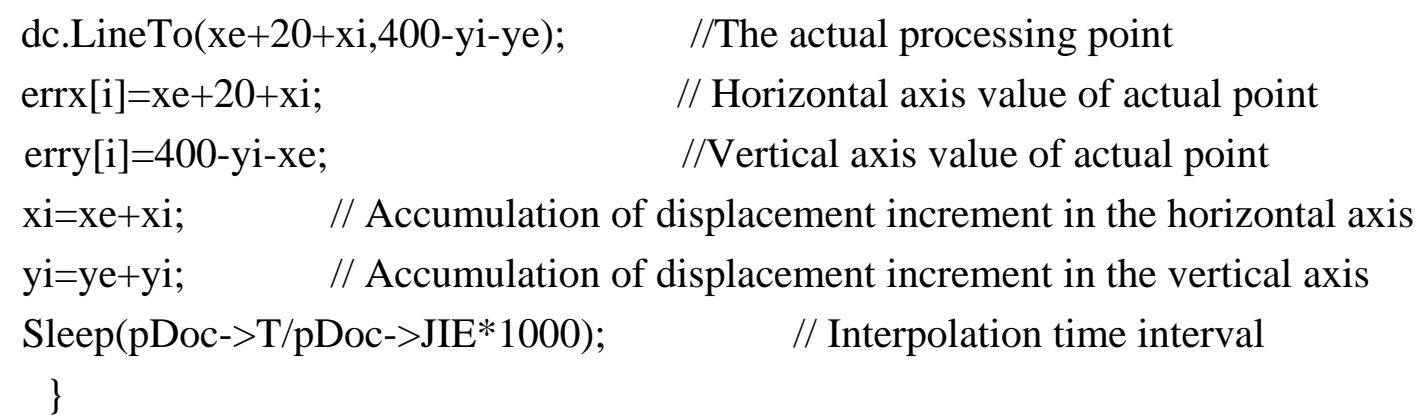

The algebraic expression (-1+rand ()\%3) *0.05 error value means caused by the stepper motor , this error is set to plus or minus $5 \%$.

Drawing correction graphic code:

void CSpdView::OnCorrect() // Draw the correction parabola

\{

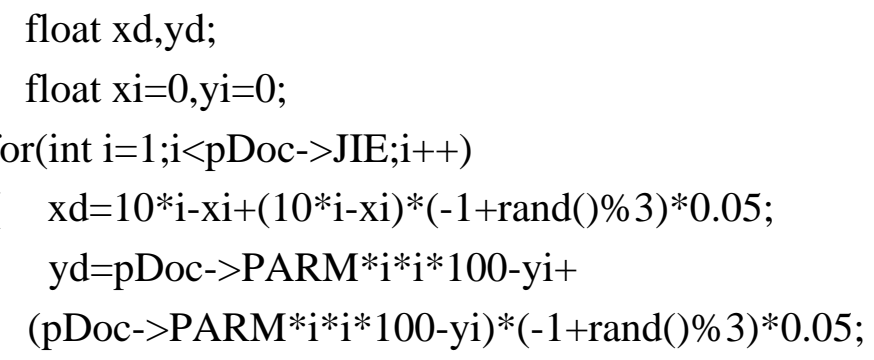

//actual displacement increment after correction

dc.LineTo(xd+20+xi,-yi-yd+400); //Reach correction point

$\operatorname{corx}[\mathrm{i}]=\mathrm{xd}+20+\mathrm{xi}$; // Horizontal axis value of correction point 


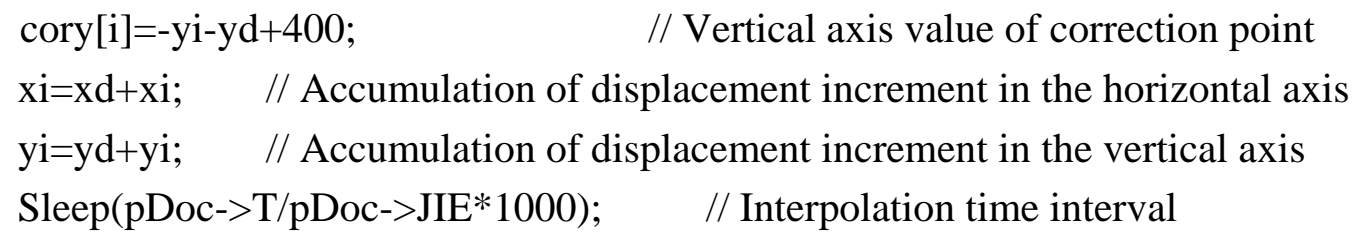

Select the graphics menu, two kinds of processing trajectory are simulated respectively. The background data processing results are obtained by pressing the buttons. As shown in Fig.4, from the graphical point of view, the error path is deviated from the theoretical path , and there are fluctuations, while the correction path is more like theoretical graphic. From the background data analysis, the standard deviation is 1.48 , the maximum deviation is 3.03 , while the standard deviation is 0.05 and the maximum deviation is 0.10 . The simulation results show that the control scheme is feasible.

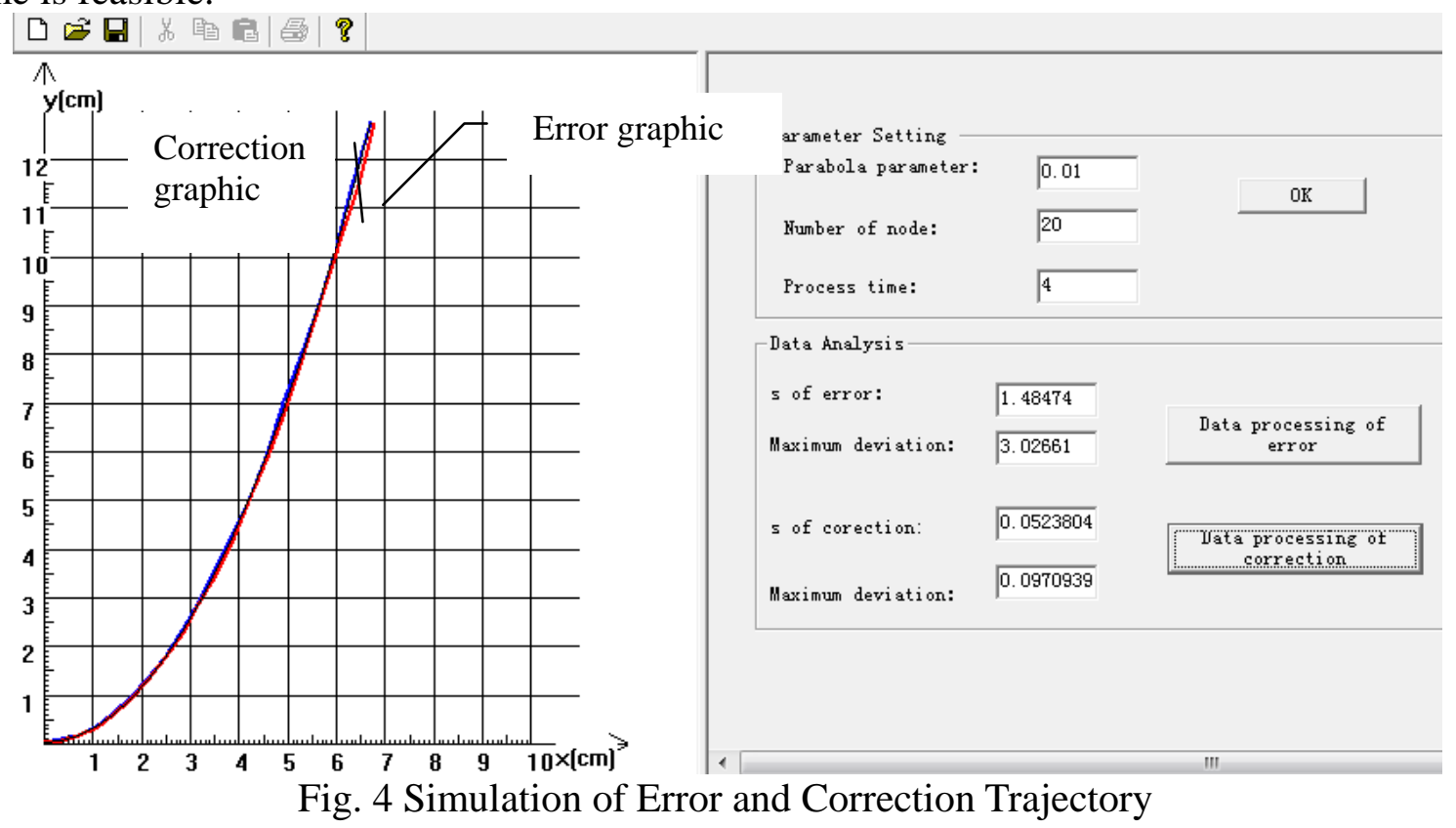

\section{Summary}

In this paper, the principle of velocity interpolation and its realization in CNC is introduced. The cause of the error in the stepper numerical control system is discussed, and the correction scheme is proposed. $\mathrm{VC}++$ software is used to simulate the process of the interpolation algorithm and the correction scheme. The feasibility of the algorithm and the control scheme is verified.

\section{References}

[1]FAN Xi-ying, Guo Yong-huan. The Development Direction of The Incremental Interpolation of NC System[J]. Machine Tools and Hydraulic, 2010,38(20):109 —111. (In Chinese)

[2] WU Hu-ping, ZHOU Ya-jun. Research on Data Sampling Interpolation Algorithm[J]. Combined Machine Tools and Automatic Processing Technology,2008,(9):13 —19. (In Chinese)

[3] YU Bo,YU Zheng-lin,GU li-dong etc. Interpolation Technology Research of CNC [J]. Acta Metrological Sinica, 2015(2), 128-130. (In Chinese)

[4]JIANG Yong-ming, CHENG Ling. Tangent Speed NC Interpolating and Its Error Study.MACHINERY,2001(1),13-16.(In Chinese)

[5]ZHOU Hong. Analysis on The Factors Affecting The Machining Accuracy of The Economic Numerical Control Machine Tools [J].2005(2):58-60. (In Chinese) 
[6]DONG Zhi-peng, HOU Jue-shu. C++ Visual Programming From Basic to Application [M].Beijing: Tsinghua University Press.2006. (In Chinese) 